\title{
Taenia solium Prevalence in Nepal: A Retrospective Review
}

\author{
B. K C* and K. Kaphle \\ Institute of Agriculture and Animal Science, Tribhuwan University, Paklihawa, Nepal \\ *Corresponding author: bishnu.kc39@gmail.com
}

\begin{abstract}
Taenia solium is a zoonotic cestode parasite which causes cysticercosis in human and porcine cysticercosis in pigs. The infective stage of $T$. solium develops in pig while that of $T$. saginata develop in cattle and buffalo. The adult stages of T. solium are obligatory intestinal parasites for man. In humans the cysticercus larvae commonly encyst in the brain, causing neurocysticercosis. The disease was first described in pig by Aristophanes and Aristotle in $3^{\text {rd }}$ century B.C. In Nepal, Taenia cysts were observed for first time in pork meat slaughtered in Kangeswari, Kathmandu more than 30 years ago. T. solium is the cause of $30 \%$ of epilepsy cases in many endemic areas where people and roaming pigs live in close proximity. More than 50 million of the world's populations are infected worldwide and 50000 die from Neurocysticercosis yearly. In Nepal, the prevalence ranges from $0.002-0.1 \%$ in general population. Accurate diagnosis required detailed post mortem examination involving slicing of affected tissue to determine the viability of cysts. Cysticerci occur most commonly in striated muscles tissues and brain of pigs. The application of vaccination with TSOL18 recombinant vaccine along with oral medication of oxfendazole can reduces the transmission by pigs whereas use of niclosamide is found effective in humans. Hence the use of medication eventually reduces the incidence of neurocysticercosis in human and procine cysticercosis in pigs.
\end{abstract}

Keywords: Cysticercosis, Pigs, Prevalence, Taenia solium

\section{INTRODUCTION}

Taenia solium is a cestode parasite of zoonotic importance which causes cysticercosis in human and porcine cysticercosis in pigs. It is a food-borne infection which is emerging as a major health problem world wide (Sciutto et.al., 2000). The infective stage of T. solium develops in pig while that of $T$. saginata develops in cattle and buffalo. The infection is endemic in the area where pigs are reared under scavenging system with poor sanitation, open defecation and uncontrolled slaughter of infected pig. It is endemic in the developing countries of Latin America, Africa and Asia (Coral-Almeida et.al., 2015). WHO has considered neurocysticercosis (NCC) as the most important neurologic disease in humans (Scheel et.al., 2005). About 50 million people of world are infected and among them 50000 die from NCC yearly (Scheel et al., 2005). Neurocysticercosis is one of the major causes of onset of symptomatic epilepsy in the areas where pork tapeworm $T$. soliumis endemic. The cysticercosis caused by $T$. solium is considered as neglected tropical disease by WHO (2015). Various control measures are operated nowadays for successful in reducing the incidence of parasite. Vaccination using TSLO18 recombinant vaccine along with oral treatment of oxfendazole @ $30 \mathrm{mg}$ per kg is found to be effective (Poudel et.al., 2019). The main aim of this paper is to know the status of T. solium by 
reviewing various articles through PubMed, Google scholars and research gate. The overall prevalence of Taenia solium in Nepal was determined along with its public health significance and proper suggestion was given to control its prevalence through rational use of vaccination and medication in both animals as well as in humans.

\section{DISTRIBUTION AND EPIDEMIOLOGY}

Taenia solium is distributed throughout the world and is particularly prevalent in the areas where small scale pig farming or pigs as household livestock is common (García et.al., 2003). Therefore, the developing countries such as Latin America, Africa, and Asia are considered as endemic areas for tapeworm. Until recent years, there were only sporadic cases in the United States. However, due to increased immigration from Central America and travel to endemic areas, cysticercosis is on the rise in the United States, especially in California, Arizona, New Mexico, Texas, and Colorado. Different forms of the disease occur in different areas depending on type and location of infection, including autoinfection or by the fecal-oral pathway. The disease cysticercosis in pigs was first discovered by Aristophanes and Aristotle during $3^{\text {rd }}$ century B.C. (Cook, 1988). Although accurate information on prevalence rates is rarely available, the disease is known to be endemic in all continents with the exception of Australasia. Exceptionally high prevalence rate occurs in Latin America from Mexico to Chile. In Mexico City 1.4-3.6\% of autopsies in general population provide evidence of cysticercosis (Cook, 1988). The disease spreads sporadically throughout the Europe and it is also prevalent in India, Pakistan, Indonesia, Thailand and northern China.

In Nepal, Taenia cyst were first observed more than thirty years ago in the pork meat slaughtered in Kangeswari, Kathmandu Joshi et.al., 2007). Neurocysticercosis was reported in Nepal during December, 1997 along with its mode of infection (Joshi et.al., 2007). Del Brutto et.al., reported that neurocysticercosis is a common cause of epilepsy among the Nepalese soldiers stationed with British Army in Hong Kong. The available data suggest that the prevalence ranges from $0.002-0.1 \%$ in general population in Nepal (Bista, 2006).

\section{LIFECYCLE}

The completion for the lifecycle of $T$. solium requires two hosts, man as definitive host and pigs as intermediate host.Pigs harbour the infection due to consumption of vegetation contaminated by the parasite's eggs or gravid proglottids. The parasites then mature to oncospheres (embryos) in the pig's intestine, which break the intestinal wall and develop into infective cysticerci (larvae) in the muscles. Infection is spread to humans when they eat raw or undercooked, infected pork meat contacting infective cysticerci larva. The larva migrates to small intestine which then matures to adult tapeworm resulting to cause taeniasis. After the parasitic larval lodges in the brain, it causes neurocysticercosis. Completion of life cycle of the parasite has been prevented in many developed countries through improvements in sanitation and hygienic practices in pig rearing, which eventually prevent the animals from being exposed to human faeces. The prevention of completion of lifecycle can facilitate in reducing the prevalence as well as prevent human from exposure to disease. The presence of suitable environment for the completion of lifecycle can be one of the causes of prevalence of this parasite in the nation. 


\section{CURRENT SITUATION IN NEPAL}

Various researches are carried out in Nepal regarding to the prevalence of $T$. solium Nepal and among them very few authentic research articles have been published till date. The published and unpublished data suggests that the disease has been established in the country, and is developing a serious public health problem.

Joshi (1973 and 1991) observed Taenia cysts in pig meat slaughtered in Kangeswari, Kathmandu for the first time more than 30 years ago. Likewise, (Bajaj, Satish K. 1997) reported about the cysticercosis and its mode of infection. Different intervention programmes are carried out in order to control its transmission. The applied intervention consists of intensive combination of mass human tapeworm deworming with niclosamide along with pig anthelmintic treatment with oxfendazole and pig immunization with the TSOL18 vaccine, in various rounds for approximately one year (Poudel et.al., 2019). In Nepal, generally poor and marginalized people are prone to infection due to close contact with pigs, poor sanitation.

Table 1: Prevalence of $T$. solium in pigs of Nepal

\begin{tabular}{|l|l|l|l|}
\hline District & No. of tested animals & $\begin{array}{l}\text { Percentage of positive } \\
\text { animals }\end{array}$ & Reference \\
\hline Banke & 110 & $32(29 \%)$ & (Sah et.al., 2017) \\
\hline Syangja & 180 & $77(43 \%)$ & (Maharjan and Gaihre, 2010) \\
\hline Tanahun & 152 & $28(18 \%)$ & (Joshi et.al., 2004) \\
\hline Kathmandu & 196 & $28(14 \%)$ & (Devleesschauwer et.al., 2013) \\
\hline Dharan & 54 & $6(11 \%)$ & (Joshi et.al., 2004) \\
\hline
\end{tabular}

\section{TAENIASIS IN HUMAN}

T. soliumcauses two diseases when they infect the human: taeniasis and cysticercosis. Taeniasis is caused by infection of the adult tapeworm in the intestines of humans and is most often acquired by eating uncooked or poorly cooked infected pork meat.Cysticercosis causes a significant public health disorder when humans ingest parasite eggs, which liberate larvae that may settles in the nervous system, causing a serious condition called neurocysticercosis (NCC) (Carabin et.al., 2017).

Cysticercosis (CC) and neurocysticercosis (NCC) are characterized by development of cyst in the eyes, skeletal muscle, heart, or brain caused due to the ingestion of Taenia solium eggs. Symptoms can vary from the clinically mild to severe, in which epileptic seizures, hydrocephalus, or localized neural deficits predate T. solium associated fatalities. Over 20 million people in the world are estimated to be infected with either the larvae or the adult tapeworm and its wide-ranging medical, economic and social effects are felt globally (Pawlowski et.al., 2005). In 2015, the WHO Foodborne disease burden Epidemiology Reference Group identified T. solium as a leading cause of deaths from food-borne diseases, resulting in a considerable total of 2.8 million disability-adjusted life-years (DALYs) (Jansen et.al., 2018). Neurocysticercosis is considered as one of the main causes of epilepsy in the developing countries of Asia, Latin America, and Africa (Pal et.al., 2000). In Nepal, human 
neurocysticercosis is a frequently diagnosed condition where it had been estimated to be responsible for the highest burden of disease caused by a parasitic zoonosis (Devleesschauwer et.al., 2012). The cases of neurocysticercosis are reported from many hospitals of Kathmandu (Amatya and Kimula, 1999). Sixty two patients with cysticercosis were confirmed out of 23,402 biopsy cases detected at Patan hospital, Lalitpur.During the past five years, records from Bir Hospital, and Kanti Children's Hospital reported 4 and 11 cysticercosis cases in Kathmandu, respectively (Joshi et.al., 2004). The main cause of epileptic seizure is now considered as neurocysticercosis which accounts for $50 \%$ of the patient with partial seizures (Rajshekhar et.al., 2003). About 50 million people are infected worldwide and among them 50000 die from NCC yearly (Scheel et.al., 2005).

\section{DIAGNOSIS}

For the proper diagnosis of $T$. solium cyst in pork meat, the pig should be slaughtered, viscera should be separated and heart, liver, brain as well as half the body skeletal musculature should be sliced using hand knives in order to determine the viability of cysts. If one or more viable or nonviable cysticerci were found in the muscle and or the brain, the pig should be confirmed of porcine cysticercosis (Poudel et.al., 2019). The case of taeniasis can also be confirmed by findings eggs or gravid proglottids in direct fecal smear. Palpation of the whole body for the presence of subcutaneous and intramuscular nodules along with neuroimaging techniques such as CT scan and MRI can also be done for diagnostic approaches in humans (Scheel et.al., 2005).

\section{TREATMENT}

Treatment of taeniasis is a good option due to direct causal link between taeniasis and human cysticercosis. For the proper treatment of taeniasis, pig should be vaccinated with $1 \mathrm{ml}$ Tsol18 vaccine $(150 \mu \mathrm{g}$ TSOL18 recombinant protein in mineral oil adjuvant; Cysvax, Indian Immunological Limited, India) through intramuscular route (Flisser et.al., 2004). Along with this intensive combination of mass human tapeworm deworming with niclosamide @ $\mathrm{g}$ as a single dose for adults along with pig anthelmintic treatment with oxfendazole @30 mg per kg body weight (Paranthic 10\%, MCI, Morocco) is found to be effective (Poudel et.al., 2019).

\section{PREVENTION AND CONTROL}

To improve $T$. solium control, we must train meat producers sellers in the safe handling of meat. Slaughtering of pigs in open space should be prohibited. The meat should be properly inspected by meat inspector before selling to market. Pig should be reared separately away from human settlement which reduces the contamination between human and pig. People must consume wellcooked as well as refrigerated meat. Sanitation and pig husbandry around the house should be properly maintained. The pigs should be immunized to control the transmission of disease. Regular and active surveillance should be done to know the prevalence and incidence of taeniasis and cysticercosis in both definitive and intermediate hosts. 


\section{DISCUSSION}

The prevalence of T.solium in Nepal is found to be significantly different according to different research conducted at different region. The highest prevalence of procine cysticercosis was recorded in Syangja district where 77 out of 180 animals were found to have cysticercosis (Maharjan and Gaihre, 2010). The lowest prevalence was recorded from Dharan district where 6 out of $54(11 \%)$ animals were found to have cysticercosis (Joshi et.al., 2004). In Banke district 32 out of $110(29 \%)$ animals were identified with T.soliumcysticerci (Sah et.al., 2017) whereas in Kathmandu 28 out of 196 (14\%) (Devleesschauwer et.al., 2013) were found to be infected. Likewise, in Tanahun 28 out of $152(18 \%)$ were found to harbour cysticercosis. These data are based on the detailed post mortem examination of carcass. These data suggest that the prevalence of cysticercosis is emerging day by day in Nepal. This is mainly due to poor sanitation, unhygienic management, close contact between pigs and animal, open defecation by humans, consumption of raw or poorly cooked pork meat. It is due to unhealthy rearing practices, where pigs are allowed to feed on human feces, lack of latrines, poor hygiene habits, and lack of meat inspection, has contributed to the prevalence of the disease. The people of terai are found rearing pig under scavenging system which initiates the cause of disease due to free roaming of animals; they transmit disease from one place to another.

The high prevalence in Syangja might be due to high number of pork consuming community such as magar, dalits etc. who rear pigs under free roaming system with poor sanitary methods (Maharjan and Gaihre, 2010). Moreover, lack of education, poor hygiene and low socio economic status may lead to its prevalence in high number.

The prevalence of porcine cysticercosis in pigs from the Banke District of Nepal indicates that there is a high rate of $T$. solium transmission in the region and, very likely to have a high rate of human cysticercosis (Poudel et.al., 2019). The unhealthy pork rearing practices, open defecation, poor hygienic habits have contributed to the establishment and transmission of the disease in human as well. The high incidence in human may due to improper eating habits of pork meat in the form of barbeque.

The reduction of prevalence of procine cysticercosis can be done by combined use of vaccine Tsol18 and administration of oxfendazole @ $30 \mathrm{mg} / \mathrm{kg}$ body weight (Poudel et.al., 2019).The combined use provides both treatment as well as prevention from subsequent infection. The drug oxfendazole helps in reduction of cysticerci whereas vaccine protects from being exposure to cysticercosis.

\section{CONCLUSION}

The review of prevalence taeniasis and cysticercosis research in Nepal showed that parasitic infections have existed for many years. The zoonotic parasite is deeply rooted in the nation which is found unnoticed due to lack of sufficient research. If this condition goes one then it will develop hazardous problem in both human as well as pig. The available data and reported cases suggest that the disease is found to be endemic in the nation which is developing into serious public health problem. Many communities who rear pig for subsistence use are unaware of 
zoonotic disease, proper sanitation and hygienic practices. Provision of public awareness should be conducted in those communities. Timely use of vaccination and medication will support for the reduction of incidence in both human as well as pigs.

\section{ACKNOWLEDGEMENT}

I am very grateful and express my gratitude to Dr. Krishna Kaphle for his constructive criticism and guidance in completing this article. I would also like to acknowledge my seniors Ram Kumar Lamsal, Dr. Sanjay Poudel, Dr. Manish Gautam, my friends Prajjwal Shrestha, Deepak Subedi, Suman Bhandari, Swochhal Prakash Shrestha and all the helping hands who assist during reviewing this article.

\section{Author's Contribution}

BKCreviewed the literature. KK provided guidance and logistic support to collect the articles. Those data were presented systematically and discussed about the reason behind the prevalence in Nepal.

\section{REFERENCES}

Amatya, B. M., and Kimula, Y. (1999). Cysticercosis in Nepal: a histopathologic study of sixtytwo cases. The American journal of surgical pathology, 23 (10), 1276.

Bista, M. (2006). Epidemiology, prevention and control of human cysticercosis/taeniasis in Nepal. Proceedings of present situation challenges in treatment and elimination of Taeniasis/cysticercosis in Nepal, edited by Joshi DD, Sharma M, Rana S. National Zoonoses and Food Hygeine Research Centre, Kathmandu, 12-14.

Carabin, H., Winkler, A. S., and Dorny, P. (2017). Taenia solium cysticercosis and taeniosis: Achievements from the past 10 years and the way forward. PLoS neglected tropical diseases, 11 (4), e0005478.

Cook, G. (1988). Neurocysticercosis: parasitology, clinical presentation, diagnosis, and recent advances in management. In: Oxford University Press.

Coral-Almeida, M., Gabriël, S., Abatih, E. N., Praet, N., Benitez, W., and Dorny, P. (2015). Taenia solium human cysticercosis: a systematic review of sero-epidemiological data from endemic zones around the world. PLoS neglected tropical diseases, 9 (7), e0003919.

Del Brutto, O., Sotelo, J., and Roman, G. (1998). Neurocysticercosis: a clinical handbook Swets and Zeitlinger. Lisse The Netherlands, 207.

Devleesschauwer, B., Aryal, A., Joshi, D. D., Rijal, S., Sherchand, J. B., Praet, N., . . Dorny, P. (2012). Epidemiology of Taenia solium in Nepal: is it influenced by the social characteristics of the population and the presence of Taenia asiatica? Tropical Medicine and International Health, 17 (8), 1019-1022.

Devleesschauwer, B., Pruvot, M., Joshi, D. D., De Craeye, S., Jennes, M., Ale, A., .. . Victor, B. (2013). Seroprevalence of zoonotic parasites in pigs slaughtered in the Kathmandu Valley of Nepal. Vector-Borne and Zoonotic Diseases, 13 (12), 872-876.

Flisser, A., Gauci, C. G., Zoli, A., Martinez-Ocana, J., Garza-Rodriguez, A., DominguezAlpizar, J. L., Aguilar-Vega, L. (2004). Induction of protection against porcine 
cysticercosis by vaccination with recombinant oncosphere antigens. Infection and Immunity, 72 (9), 5292-5297.

García, H. H., Gonzalez, A. E., Evans, C. A., Gilman, R. H., and Peru, C. W. G. i. (2003). Taenia solium cysticercosis. The lancet, 362 (9383), 547-556.

Jansen, F., Dorny, P., Trevisan, C., Dermauw, V., Laranjo-González, M., Allepuz, A., . . . Devleesschauwer, B. (2018). Economic impact of bovine cysticercosis and taeniosis caused by Taenica saginata in Belgium. Parasites and vectors, 11 (1), 241.

Joshi, D., Poudyal, P. M., Neave, M. J. L., and Maharjan, M. (2007). Epidemiological status of Taenia/cysticercosis in pigs and human in Nepal. Journal of Institute of Medicine, 23 (1).

Joshi, D. D., Bista, P. R., Ito, A., and Yamasaki, H. (2007). Present situation of porcine taeniasis and human cysticercosis in Nepal. Southeast Asian journal of tropical medicine and public health, 38 (1), 144.

Joshi, D. D., Maharjan, M., Johnsen, M. V., Willingham, A. L., Gaihr, Y., and Sharma, M. (2004). Taeniasis/cysticercosis situation in Nepal. Southeast Asian J Trop Med Public Health, 35 (1), 252-258.

Maharjan, M., and Gaihre, Y. K. (2010). Porcine Cysticercosis in the Magar Community of Syangja District. Lifesci. Journo-Mag., 2, 20-26.

Organization, W. H. (2015). Investing to overcome the global impact of neglected tropical diseases: third WHO report on neglected tropical diseases 2015 (Vol. 3): World Health Organization.

Pal, D. K., Carpio, A., and Sander, J. W. (2000). Neurocysticercosis and epilepsy in developing countries. Journal of Neurology, Neurosurgery and Psychiatry, 68 (2), 137-143.

Pawlowski, Z., Allan, J., and Sarti, E. (2005). Control of Taenia solium taeniasis/cysticercosis: from research towards implementation. International journal for parasitology, 35 (1112), 1221-1232.

Poudel, I., Sah, K., Subedi, S., Singh, D. K., Kushwaha, P., Colston, A., . . . Lightowlers, M. W. (2019). Implementation of a practical and effective pilot intervention against transmission of Taenia solium by pigs in the Banke district of Nepal. PLoS neglected tropical diseases, 13 (2), e0006838.

Rajshekhar, V., Joshi, D. D., Doanh, N. Q., van De, N., and Xiaonong, Z. (2003). Taenia solium taeniosis/cysticercosis in Asia: epidemiology, impact and issues. Acta tropica, 87 (1), 5360.

Sah, K., Poudel, I., Subedi, S., Singh, D. K., Cocker, J., Kushwaha, P., . . Lightowlers, M. W. (2017). A hyperendemic focus of Taenia solium transmission in the Banke District of Nepal. Acta tropica, 176, 78-82.

Scheel, C. M., Khan, A., Hancock, K., Garcia, H. H., Gonzalez, A. E., Gilman, R. H., and Tsang, V. C. (2005). Serodiagnosis of neurocysticercosis using synthetic 8-kD proteins: comparison of assay formats. The American journal of tropical medicine and hygiene, 73 (4), 771-776.

Sciutto, E., Fragoso, G., Fleury, A., Laclette, J. P., Sotelo, J., Aluja, A., . . Larralde, C. (2000). Taenia solium disease in humans and pigs: an ancient parasitosis disease rooted in developing countries and emerging as a major health problem of global dimensions. Microbes and infection, 2 (15), 1875-1890. 